was hardly perceptible; the body was bathed in sweat. It was evident to all that he was fast sinking; and he died at about 11 o'clock, five days after the first appearance of the vesicle.

The general treatment consisted chiefly in the liberal administration of good beef-tea, brandy, and ammonia.

[To be continued.]

\section{OHriginal Commanications.}

\section{DISLOCATION OF THE KNEE.JOINT.}

By T. Ogier Wand, M.D., Cowbridge.

As dislocations of the knee-joint are rare, I send an account of one in which reduction was effected by flexion instead of extension, the latter having completely failed.

A young gentleman, aged 15 , riding a velocipede down a steep hill, was thrown off, and fell on his left knee. He walked home, and four hours afterwards came to me, when I found the knee very much swelled from effusion into the joint, the patella projecting about an inch, and half an inch lower than the right from the tension of the ligament. The limb was straight, and could not be flexed without pain, though painless when quiet and extended. A side view of the limb showed little difference between the left and right behind; but in front the prominence of the patella clearly proved that the tibia was dislocated backwards. There was a bruise and abrasion of the skin over the internal condyle. The limb was not shortened; indeed, if at all, it was longer than the other.

Thinking that, with so little change in the form of the limb, the reduction by extension would not be diffi. cult, I first tried that plan; but, though I put the patient under the influence of chloroform, and gave him several half-grain doses of tartar emetic, and had two strong men making extension with a roller-towel round the ankle, at the same time that $I$ was trying to push the head of the tibia forwards, not the least effect was produced. I then recollected that I had formerly reduced a similar case, where the patella was fractured, by flexion of the joint over the knee of an assistant: so, taking the towel off the ankle, and thus diverting the attention of the patient, I suddenly pushed the heel backwards till it touched the thigh, when the reduction was completed, and the power of flexion was restored. I then bound a slight splint round the extended limb; and, with the aid of cold lotions, in a few days the effusion was absorbed; and in three weeks the cure was completed, and the bandage was removed.

The rarity of this form of dislocation, and other pecu liarities in the symptoms and treatment, suggest a few remarks. I have before me the descriptions of two of the most recent writers on this subject, Mr. Erichsen and Mr. Lane. The former states (Science and Art of Surgery, 3rd edition, pp. 290-291): "The dislocation backwards (of the tibia) may be complete or incomplete. When it is complete, the posterior ligament of the joint is torn, the muscles of the ham are stretched, the limb is shortened to the extent of an inch and a half or two inches, and is semiflexed; the head of the tibia can be felt in the ham, and there is a deep transverse depression in front of the joint immediately below the patella. ... The antero-posterior dislocations are very commonly incomplete. When this is the case, they present the same symptoms, but in a less marked degree, that characterise the complete displacements." In treatment of dislocations of the knee, Erichsen recommends "extension from the ankle while the thigh is fixed in a semiflexed position".
In this account, the possibility of the limb being lengthened is overlooked; and the treatment is the same as has been recommerded by all previous writers-viz. extension of the affected limb.

Mr. J. Lane also (Cooper's Surgical Dictionary, 1861, article Dislocations), quoting Malgaigne, omits all mention of the lengthening of the limb; hut to the recommendation of extension, Lane adds: "Should any difficulty occur after the necessary extension has been made, the effect of flexing the knee may be tried; or the knee may be bent round the bedpost, or over the left arm or knee of the operator." He makes, however, no distinction as to the class in which flexion is best.

In describing the incomplete luxation backwards, Mr. Lane, or Malgaigne, asserts that "the limb is sometimes extended, sometimes slightly flexed," which latter position soems impossible, unless the ligamentum patellæ be ruptured; and therefore, I think, in this respect he has confounded the dislocation of the semilunar carti. lages (of which, slight flexion with inability to extend the knee, is the pathognomonic symptom) with incomplete luxation of the tibia backwards. Sir Astley Cooper's description, omitting the shortening of the limb, is most characteristic-" a projection of the condyles of the femur, a depression of the ligamentum patellæ, and a bending of the leg forwards"; but he also recommends only extension for the reduction.

My own case agreed with Sir A. Cooper's description, except as regards the shortening of the limb and depression of the patella ; which, however, might be said to be depressed at its lower end under the extremity of the femur, while the upper part was raised by the effusion beneath and the tilting up produced by the depression of the other end. In this, as in the former case I have alluded to, the nature of the injury was evident from the bending of the leg forward; and yet in neither instance could reduction be made by extension, though it was readily effected by flexion of the limb. How are these discrepancies in the treatment and the shortening of the limb to be reconciled? Only, I think, by considering the cases requiring flexion for their reduction as incom. plete luxations, and those reduced by extension as complete luxations attended with rupture of the ligaments. This view will explain the facility of the reduction of the latter (for, the ligaments being ruptured, there would be less power of resistance to the extension); and, vice versá, the very great difficulty, if not impossibility, of the reduction by extension when the luxation was slight and the ligaments uninjured. In proof of this difficulty, I recollect seeing $\mathrm{a}$ boy of 15 , who had dis. located the similunar cartilages, resist with ease the united efforts of six persons to straighten his knee, which, as usual in that accident, was bent, though the power to straighten it returned after a few days rest.* In the last case of execution in France by the victim being torn ssunder by four horses, each attached to a limb, the utmost efforts of the animals failed, until the ligaments had been divided.

It is hardly necessary to state that luxations not attended with rupture of the ligaments would require very little treatment after reduction. Again, the reduction of any case by flexion necessarily implies only a slight displacement of the ends of the bones; for, if the head of the tibia were pushed far backwards, flexion could not be effected without fracture of the patella or rupture of its ligament. The slight displacement may also explain the absence of shortening of the limb, and account for its being lengthened; for, if the condyles of the femur were thrown upon, and rested on the anterior tuberosities of the tibia, instead of on the hollow sur.

* Thongh Sir A. Cooper recommends flexion of the knee in dislo cation of the semilunar cartilages, reduction will take place spon taneously in $n$ few days by the gradual stretching of the tissue con necting them with the capsular ligament, and thus a painful opera tion may be avoided. 
faces behind them, the limb must necessarily be lengthened.

If these statements and arguments are correct, it seems to be proved that, in incomplete luxations of the tibia backwards, flexion, and not extension, is the easiest mode of reduction, and that in such cases the limb may be lengthened.

P.S. As there seems to be a question respecting the influence of bleeding or loss of blood in the production of softening of the brain, I may mention that $I$ was re. cently called in to a woman who had a "fit" on the ninth day after a very easy labour, with very little loss of blood. I found her slightly paralysed in the right hand and arm, but unable to speak plainly, or to find the right word to express her meaning. The left pupil was natural, perhaps a little dilated; but the right was very much contracted. Since the attack the previous night, she had vomited, and had diarrhœa, the last mo. tion being bloody mucus. I ordered her a chalk mixture with landanum, the first dose of which stopped the vomiting and diarrhoa. The next (third) day she com plained of pain in the left side of her head; and I ordered four leeches, which were not applied till the night of the fourth day, when only two drew blood, at the outer angle of the eye, about six o'clock. At 11 P.x. she had another fit, became insensible, and remained so tili her death, twenty-four hours after the attack, with occasional convulsive movements of the affected arm. I saw her twelve hours after the attack, when the eyes presented the same appearance. I did not examine the head; but the symptoms clearly point to softening of the brain, rather than apoplexy; and I cannot believe that the small quantity of blood drawn by two leeches could have caused a recurrence of the "fit". Her eldest son has epileptic fits; and I am disposed to attribute the mother's disease to perhaps a tendency to disease of the brain, increased by grief at the recent death of her father, and brought to a climax by the straining of even an easy labour. The absence of pain till after the fit is accounted for by the insensibility of the cerebral sub. stance.

\section{CASE OF PELLAGRA.}

By Sayuel Wilks, M.D.

THE observations of Dr. Peacock on pellagra, contained in the last number of the NIedico.Chirurgical Review, have again brought before my mind a case which $I$ witnessed last autumn, and which I suggested might be an example of an affection which is almost peculiar to the north of Italy. No English author that I am aware of, has ever described such a form of disease in this country, and, therefore, it is with considerable dif. fidence that $I$ bring forward the present case as an example of it; at the same time the case so much resembles the descriptions which I had read of this remarkable malady, that I informed the medical man under whose care the patient had been, that I could make nothing of it unless it were a case of pellagra. The report of the case, althnugh brief, may be the means of drawing some similar instance from the note-books of other practi. tioners. I believe it to be true that many diseases which are rife in some countries, and almost peculiar to them, may occur in isolated instances, und in modified characters, in other parts of the world; and, thus it is that elephentiasis or leprosy may be sometimes met with in our own temperate island. In the same way, pellagra, which is described by French writers as not occurring in Paris, yet was supposed to exist in the persons of four patients which were in La Charité scome years ago. (M. Willemin in Archives Générales de Médecine, Series 4, vol. xiii.)

The disease, as it is witnessed in Italy, is an affection of a very chronic nature, continuing for several years, characterised by a general wasting of the body, and by a rash which covers the exposed parts of the skin, commencing as an erythema, and progressing into a lichenous or eczematous eruption. At a later period, sores sometimes appear, impairment of digestion, and nervous symptoms, a paralysis of the limbs. The cause of the disease appears at present not decided, it having been thought by some that it is due to exposure to the sun, from the fact of exacerbations occurring in the summer time; by others, that it is due merely to poverty, as it does not prevail amongst the rich; but these and similar supposed theories, are refuted by the remembrance that such causes are not peculiar to Lombardy, but exist where pellagra is quite unknown. The most recent theory places the cause in the consumption of diseased maize, the corn being affected somewhat after the manner of the ergotised rye.

In the four cases which occurred at Paris, described by M. Willemin, and which appeared to be true examples of the disease, the skin was covered either with an erythematous, a lichenous, or eczematous rash. This was on the face and arms, and had a thickly defined margin; in two cases a diarrhœa existed; in one the gums were spongy, as in scurvy, and in some there was a weakness of the extremities. The pulse was feeble in all, and in one case, after the patient bad been lying down, was reduced to thirty beats in the minute.

The peculiarities of the disease appear to be: in the first place, this cutaneous eruption, which is remarkable in affecting the parts exposed to the light, as the face, neck, chest, backs of hands, arms, or feet, the redness ceasing abruptly at a line where the covering of the clothes commences; the skin also becoming hard and dry like parchment, and in some cases bullæ appearing. Next in imporiance to the cutaneous rash, are various nervous symptoms, as numbness or pains in the limbs, and sometimes a veritable paralysis, as in myeitis of the spinal cord. It is said by some writers that in very bad examples of the disease, a melancholy overtakes the patient determining to suicide, especially by drowning, and to this has been given the special name of hydromania. Other writers, however, are ignorant of the existence of this propensity. As regards the pathology of the disease, nothing is known; some have regarded it as a chronic gastro-enteritis; whilst others, at a loss to discover the seat of the disease, have been content to style it morbus chronicus totius corporis.

The case which came before me last autumn, was that of a woman, living at Glastonbury, and intimately known to $\mathrm{Mr}$. Malton of that place. This gentleman sent the case up to his relative Dr. Charlton of Dartford, who again brought her to me for an opinion as to the nature of her complaint, the obscurity of which suggested to the former gentleman, I apprehend, that there might be some disease of the suprarenal capsules. She was married, aged 33, and had three children. She informed me that she had been ill for six years, having become very thin and being covered in parts with an eruption. She said that her illness had commenced five and a half years before, in the month of May, after exposure to the sun. These statements were not extracted from her by leading questions; for it was not until after she had left my house, and I was revolving the case in my mind, that the idea struck me, that it was one of pellagra. Soon after this exposure to the sun, a rash appeared on the face and arms, and subsequently she lost flesh and became weak. This rash, she said, had continued ever since with varied severity, being aggravated in summer, and better in winter. Her general appearance was very remarkable, presenting as she did a withered and emaciated look, reminding one of a child in the last.stage of marasmus; all the outlines of the face were very prominent, the cheeks were sunken in and the temporal muscles much wasted. The face was covered with a 